\title{
Lese-Rechtschreib-Schwäche
}

\section{Ein Leben für die Legasthenietherapie}

\author{
Mit großem Engagement hat Edith-Maria Soremba fast ihr ganzes \\ Leben dem Thema Legasthenie gewidmet. Vielen betroffenen \\ Kindern und auch Erwachsenen konnte sie als Grundschullehrerin \\ und später als Legasthenie-Therapeutin helfen. Was sie bewegt, \\ wofür sie kämpft und was ihre Visionen sind, darüber unterhielten \\ wir uns mit der temperamentvollen alten Dame.
}

? Wie kamen Sie dazu, sich mit dem Thema Lese-Rechtschreib-Schwäche zu befassen?

Soremba: Ich bin 1967 als Grundschullehrerin auf dieses Problem gestoßen, da hatte ich das Schlüsselerlebnis meines Lebens: Ich unterrichtete damals an einer kleinen Landschule, als mir ein Junge geschickt wurde, der buchstäblich überhaupt nicht lesen konnte. Ich war fassungslos! Ein Schwerstfall von Legasthenie. In der Klasse des Kindes, das aus einer kinderreichen Familie stammte, gab es oft Ohrfeigen als Erziehungsmaßnahmen; der Junge litt furchtbar. Dabei war er sonst begabt und verständig. Ich habe ihm Liebe und Verständnis gegeben, mit dem damaligen Kenntnisstand - man wusste rein gar nichts über Legasthenie - konnte ich ihm aber nicht helfen.

Trotz meiner Einwände bekam der Junge am Ende der achten Klasse kein Abschluss-, sondern nur ein Abgangszeugnis, obwohl er gut durch Leistungen in anderen Fächern hätte ausgleichen können. Dennoch fand er Arbeit, aber die Geschichte hat ein schreckliches Ende gefunden: Der Junge kaufte sich ein Mofa, er übersah an einer Kreuzung die Verkehrszeichen und verunglückte tödlich. Ich bin überzeugt, dass ein optisches Wahrnehmungsproblem Ursache für den schrecklichen Unfall war, der sich mir stark eingeprägt hat.

? Sie waren in gewisser Weise Pionierin in Sachen Legasthenie?

Soremba: Das kann man wohl so sagen. Als die ersten Berichte zu dem Thema an die breite Öffentlichkeit gelangten, be- schäftigte ich mich intensiv damit. Bald wurde ich neben vielen anderen Kollegen und Kolleginnen zur Legasthenie-Obmännin ausgebildet. Dass auch begabte Kinder nicht lesen und schreiben können - das war für viele damals ein Schreckgespenst, von dem man nicht recht glauben wollte, dass so etwas auch an deutschen Schulen existiert. Bei meinem Eintreten für die Belange der Betroffenen wurde ich oft auch angegriffen. Das hat mich aber nicht eingeschüchtert, ich habe weiter an die Verantwortlichen appelliert und am Ende ja zusammen mit allen Mitstreitern der Anerkennung der Legasthenie als reales, ernst zu nehmendes Problem mit zum Durchbruch verholfen.So habe ich auch einmal dem Kultusminister geschrieben und ihm erklärt, dass man normale Rechtschreibfehler nicht mit Fehlern in einen Topf werfen kann, die aufWahrnehmungsstörungen, Lautnuancentaubheit oder Raumlagelabilität beruhen. Heute gibt es ja entsprechende Erlasse, die dies anerkennen.

\section{? Was machen Sie heute?}

Soremba: Auch mit meinen 83 Jahren ist mir das Thema Legasthenie immer noch sehr wichtig: Ich arbeite weiter mit meinem Sohn in unserer Praxis für integrative Lerntherapie, die traditionell überfüllt ist. Außerdem biete ich ehrenamtlich jeden Abend zwischen 20 und 21 Uhr einen unentgeltlichen Beratungsdienst an, für Betroffene, Angehörige, Ärzte und Lehrer. Das wird sehr dankbar angenommen. Wir sind mit unserer Arbeit noch lange nicht am Ende. Und dann schreibe ich gerade an meinem fünf-

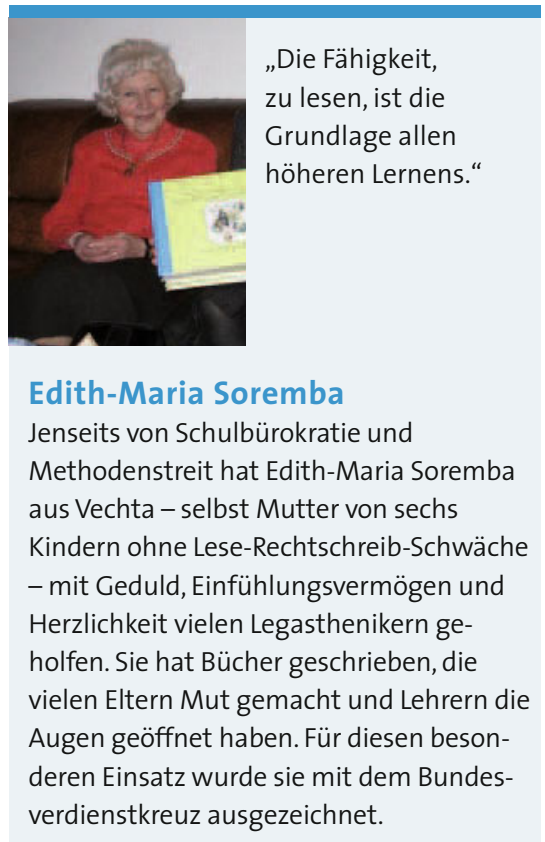

ten Buch mit dem vorgesehenen Titel „Papa, ich will lesen lernen!“

? Gibt es aus Ihrer Erfahrung überhaupt die Legasthenie - oder hat man es möglicherweise mit einer Vielzahl ganz unterschiedlicher, individueller Störungen zu tun?

Soremba: Natürlich gibt es die Legasthenie. Ganz grob skizziert ist das eine Schwäche beim Erlernen des Lesens und Schreibens, ohne Beeinträchtigung der intellektuellen Leistungsfähigkeit und ohne Fehler oder Fehlverhalten seitens des Kindes, der Eltern oder der Schule - in der Regel jedenfalls.

Ein Legastheniker behält diese Schwäche bis an sein Lebensende. Ich sehe das immer wieder auch bei Erwachsenen, oft hochbegabten Menschen, übrigens gerade auch bei Ärzten und allgemein bei Naturwissenschaftlern, kreativen Menschen - Menschen, die wir brauchen! Und dass solche Leute nicht schon als Kleine aussortiert werden, dafür kämpfe ich! 
? Die gesellschaftlichen und schulpolitischen Rahmenbedingungen für Legastheniker haben sich geändert. Würden Sie rückblickend sagen, dass die Schwierigkeiten Betroffener heute früher als Störung erkannt werden und die Legasthenie im Schulbetrieb eher akzeptiert wird?

Soremba: Jein: die höheren Schulen sind oft aufgeschlossener als die Grundschule. Dabei ist diese ja verantwortlich für so vieles: Sie prägt die Lernbiografie - was in dieser Zeit versäumt wird, ist kaum wieder gutzumachen. Die Gymnasien arbeiten meistens ganz gut mit uns zusammen, wenn wir betroffenen Kindern ein Gutachten erstellen und darum bitten, ihnen Notenschutz oder Nachteilsausgleich zu gewähren.

? Was treibt Sie an?

Soremba: Für mich steht das Individuum mit seinen Nöten und Qualen im Mittelpunkt. Nach meiner Grundüberzeugung kann und darf nicht sein, dass so ein kleiner Mensch, der sich auf die Schule gefreut hat, auf einmal lernen muss, dass er ein Versager ist und unter Tränen und Seelenqualen lesen und schreiben erlernen muss! Das Selbstbewusstsein eines Kindes muss erhalten bzw. gestärkt werden - wie das eines jeden Menschen.

Oder denken Sie nur einmal an den Analphabetismus: Geschätzte vier Millionen Deutsche können nicht richtig lesen und schreiben! Viele glauben ja gar nicht, dass es das bei uns gibt.

Die Betroffenen leiden unter Schamgefühl, Ausgrenzung und Hoffnungslosigkeit. In meinem Beratungsdienst, wo sie keinen Namen zu nennen brauchen, öffnen sich viele in ihrer Seelennot.

? Was wollen Sie den Lesern in Ihrem neuen Buch zum Thema "Lesen lernen vor dem Schuleintritt"vermitteln?

Soremba: Ich möchte den Eltern sagen: Ein Kind lernt vom ersten Augenblick seines Lebens an, und was wir am Anfang versäumen, ist nachher gar nicht wieder gutzumachen! Kinder können im frühesten Alter der Sprachentwicklung das Lesen erlernen, und Lesenlernen ist entscheidend wichtig für die Entwicklung von Intelligenz und Begabung. Die Fähigkeit, zu lesen, ist die Grundlage allen höheren Lernens. Je früher wir also den Kindern Augen und Sinne zum Lesen öffnen, umso besser.

Ein Kind hat ja von Beginn an optische Lerneindrücke. Man hat herausgefunden, dass schon Säuglinge in der Lage sind, ganz bestimmte, große Schriftbilder zu erfassen, zu speichern und wieder abzurufen - nur soviel vorab zu diesen neuen Forschungserkenntnissen. Mir geht es darum, dass die Kinder mit Freude an das herangebracht werden, was ihnen später im Leben möglicherweise hilft. Wesentlich ist natürlich, dass dies mit Liebe und ohne Drill geschieht.

! Frau Soremba, vielen Dank für das Gespräch.

\section{Das Interview führte Waltraud} Paukstadt.

\section{Sprachtest jetzt auch für Dreijährige}

Der Kurztest zur Sprachbeurteilung von zweijährigen Kindern durch die Eltern, SBE-2-KT, wurde nun durch den SBE-3-KT für die Dreijährigen ergänzt. Die Fragebogentests wurden an der Poliklinik für Kinder- und Jugendpsychiatrie der Ludwig Maximilians Universität München entwickelt und können unter den folgenden Webadressen kostenlos aus dem Internet heruntergeladen werden:

\section{www.kjp.med.uni-muenchen.de/sprachstoerungen/SBE-2-KT.php} www.kjp.med.uni-muenchen.de/sprachstoerungen/SBE-3-KT.php

Auch wenn das IOWiG kürzlich den Nutzen einer frühen Diagnose und Therapie von Sprachentwicklungsverzögerungen mangels vorliegender Studien bezweifelte, sind dem Kinderarzt durch die beiden Tests Instrumente an die Hand gegeben, die eine genaue Einschätzung und Verlaufsbeurteilung der Sprachentwicklung wesentlich erleichtern. 